\title{
Optimal Manoeuver Trajectory Synthesis for Autonomous Space and Aerial Vehicles and Robots
}

\author{
Dr. Ranjan Vepa ${ }^{1}$, \\ School of Engineering and Material Science, Queen Mary, University of London, London, E14NS, UK. \\ r.vepa@qmul.ac.uk.
}

\begin{abstract}
In this paper the problem of the synthesis of optimal manoeuver trajectories for autonomous space vehicles and robots is revisited. It is shown that it is entirely feasible to construct optimal manoeuver trajectories from considerations of only the rigid body kinematics rather than the complete dynamics of the space vehicle or robot under consideration. Such an approach lends itself to several simplifications which allow the optimal angular velocity and translational velocity profiles to be constructed, purely from considerations of the body kinematic relations. In this paper the body kinematics is formulated, in general, in terms of the quaternion representation attitude and the angular velocities are considered to be the steering inputs. The optimal inputs for a typical attitude manoeuver is synthesized by solving for the states and co-states defined by a two point boundary value problem. A typical example of a space vehicle pointing problem is considered and the optimal torque inputs for the synthesis of a reference attitude trajectory and the reference trajectories are obtained.
\end{abstract}

Keywords: attitude manoeuvers, optimal manoeuver trajectory, trajectory optimization, trajectory tracking.

\section{Introduction}

The concept of using the kinematic equations of a vehicle or robot for trajectory synthesis has been around for some time, since it was introduced by several authors. (see for example Vukobratovic and Kircanski, 2013). It has been successfully used for trajectory planning of drones and car-like robots. (Lagache, Serres, and Andrieu, 2017, Wolek, Cliff and Woolsey, 2016). However for the case space vehicles and robots the use of the kinematic equations, particularly formulations based on quaternions and dual quaternions, is relatively new. Özgür and Mezouar (2016) have used a dual velocity representation to develop an expression for the Jacobian matrix and to perform kinematic control on a robotic arm. Using the dual velocity concept, Valverde and Tsiotras (2018) have extended the quaternion kinematic law to an equivalent formulation in terms of dual quaternions, after providing an introduction to them. The formulation developed by them reduces to a sequence of quaternion updates, which in some cases reduces further to a set scalar update equations. While the adaptive attitude control problem using dual quaternions has only recently been solved (Filipe and

${ }^{1}$ Corresponding author: Tel: +4420788257193; E-mail address: r.vepa@qmul.ac.uk. (Ranjan Vepa);

ORCID ID: 0000-0003-1293-0953 
Tsiotras, 2014), the corresponding attitude and translational optimal trajectory synthesis problem has not received much attention.

In this paper the problem of the synthesis of optimal manoeuver trajectories for autonomous space vehicles and robots is revisited. It is shown that it is entirely feasible to construct optimal manoeuver trajectories from considerations of the only rigid body kinematics rather than the complete dynamics of the space vehicle or robot under consideration. Such an approach lends itself to several simplifications which allow the optimal angular velocity and translational velocity profiles to be constructed, purely from considerations of the body kinematic relations. In this paper the body kinematics is formulated, in general, in terms of the quaternion representation attitude and the angular velocities are considered to be the steering inputs. The optimal inputs for a typical attitude manoeuver are synthesized by solving for the states and co-states defined by a two point boundary value problem. A typical example of a space vehicle pointing problem is considered and the optimal torque inputs for the synthesis of a reference attitude trajectory are obtained. Based on the reference trajectory and a typical tracking controller, it is shown that the reference trajectory can be successfully tracked. The approach has the added advantage that it could be generalized to deal with complex space robotic mechanisms on-board a space platform. This is done by defining decoupled attitude and orbit trajectory synthesis problems both for the vehicle and for the on-board robot manipulators.

\section{Optimal Attitude Orientation Acquisition Trajectory Synthesis}

To begin with the quaternion kinematics can be expressed in one of two alternate forms which are given as.

$$
\frac{d \mathbf{q}}{d t}=\frac{1}{2} \mathbf{A}_{\boldsymbol{\omega}}(\boldsymbol{\omega}) \mathbf{q}=\frac{1}{2} \Gamma(\mathbf{q}) \omega,
$$

where, where the quaternion $\mathbf{q}=\left[\begin{array}{llll}\varepsilon_{1} & \varepsilon_{2} & \varepsilon_{3} & \eta\end{array}\right]^{T}$, consists of a vector part, $\boldsymbol{\varepsilon}=\left[\begin{array}{lll}\varepsilon_{1} & \varepsilon_{2} & \varepsilon_{3}\end{array}\right]^{T}$ and the scalar $\eta$ so,

$$
\begin{gathered}
\mathbf{q}=\left[\begin{array}{c}
\boldsymbol{\varepsilon} \\
\eta
\end{array}\right] \text { and } \mathbf{A}_{\boldsymbol{\omega}}=\left[\begin{array}{cc}
-\boldsymbol{\Omega}(\boldsymbol{\omega}) & \boldsymbol{\omega} \\
-\boldsymbol{\omega}^{T} & 0
\end{array}\right], \Omega(\boldsymbol{\omega})=\left[\begin{array}{ccc}
0 & -\omega_{3} & \omega_{2} \\
\omega_{3} & 0 & -\omega_{1} \\
-\omega_{2} & \omega_{1} & 0
\end{array}\right] . \\
\Gamma(\mathbf{q})=\left[\begin{array}{c}
\eta \mathbf{I}_{3 \times 3}+\mathbf{S}(\boldsymbol{\varepsilon}) \\
-\boldsymbol{\varepsilon}^{T}
\end{array}\right], \mathbf{S}(\boldsymbol{\varepsilon})=\left[\begin{array}{ccc}
0 & -\varepsilon_{3} & \varepsilon_{2} \\
\varepsilon_{3} & 0 & -\varepsilon_{1} \\
-\varepsilon_{2} & \varepsilon_{1} & 0
\end{array}\right],
\end{gathered}
$$

and $\mathbf{I}_{3 \times 3}$ is the $3 \times 3$ unit matrix. Although equations (2.1) can be generalized and both the quaternion and angular velocity can be expressed as a dual quaternion and dual angular velocity vector as outlined by Sjøberg and Egeland (2018) the dual component of the quaternion satisfies an additional constraint which is not easily implemented, in practice. Thus the dual component has effectively only three independent variables and represents the translational kinematics.

In equations 2.1, the angular velocity vector is treated as a control variable and expressed as, 


$$
\boldsymbol{\omega}=|\boldsymbol{\omega}|_{\max } \boldsymbol{u},
$$

where the direction vector $\boldsymbol{u}$ is parametrized by two angles defining the direction of the $\boldsymbol{\omega}$ vector. Thus the direction vector $\boldsymbol{u}$ is expressed as,

$$
\boldsymbol{u}=\left[\begin{array}{lll}
\sin \alpha \cos \beta & \cos \alpha \cos \beta & \sin \beta
\end{array}\right]^{T} .
$$

When one is interested in the problem of finding the directional control,

$$
\boldsymbol{u}=\boldsymbol{u}(t), t_{0} \leq t \leq t_{f},
$$

the angular velocity direction time history is sought, such that it minimizes the cost functional:

$$
J=\left.0.5\left(\mathbf{q}(t)-\mathbf{q}_{d}\right)^{T} \mathbf{Q}_{f}\left(\mathbf{q}(t)-\mathbf{q}_{d}\right)\right|_{t_{t=t_{f}}}=\left.\Phi\{\mathbf{q}(t)\}\right|_{t=t_{f}},
$$

subject to, equations (6.1), (6.2) and (6.3). Introducing the single state vector, $\mathbf{x}=\mathbf{q}$ so the equations (2.1), are expressed as,

$$
d \mathbf{x} / d t=\mathbf{f} .
$$

To solve the optimization problem, three Lagrangian multipliers or co-states are introduced given by the vector $\lambda_{\mathbf{q}}(t)$. Following Bryson and Ho (1969), a Hamiltonian function is defined as,

$$
H=\lambda_{\mathbf{q}}^{T}\left(0.5 \mathbf{A}_{\omega}(\omega) \mathbf{q}\right)=\lambda_{\mathbf{q}}^{T}(0.5 \Gamma(\mathbf{q}) \boldsymbol{\omega})=\left(|\omega|_{\max } / 2\right)\left(\lambda_{\mathbf{q}}^{T} \Gamma(\mathbf{q})\right) u .
$$

The corresponding differential equations that the co-state vector must satisfy are,

$$
\frac{d}{d t} \lambda_{\mathbf{q}}^{T}(t)=-\frac{\partial H}{\partial \mathbf{q}}=-\frac{1}{2} \lambda_{\mathbf{q}}^{T} \mathbf{A}_{\boldsymbol{\omega}}(\omega)
$$

The optimality conditions for the control parameters are,

$$
\frac{\partial H}{\partial \alpha}=\frac{|\omega|_{\max }}{2} \lambda_{\mathbf{q}}^{T} \Gamma(\mathbf{q})[\cos \alpha \cos \beta \quad-\sin \alpha \cos \beta \quad 0]=0,
$$

and

$$
\frac{\partial H}{\partial \beta}=\frac{|\omega|_{\max }}{2} \lambda_{\mathbf{q}}^{T} \Gamma(\mathbf{q})\left[\begin{array}{lll}
-\sin \alpha \sin \beta & -\cos \alpha \sin \beta & \cos \beta
\end{array}\right]=0 .
$$

Hence it follows that the c-state vector $\lambda_{\mathbf{q}}(t)$, satisfies the relation,

$$
\frac{|\boldsymbol{\omega}|_{\max }}{2} \Gamma(\mathbf{q}) \lambda_{\mathbf{q}}=\left|\frac{|\boldsymbol{\omega}|_{\max }}{2} \Gamma(\mathbf{q}) \lambda_{\mathbf{q}}\right|\left[\begin{array}{lll}
\sin \alpha \cos \beta & \cos \alpha \cos \beta & \sin \beta
\end{array}\right]^{T}=\left|\frac{|\omega|_{\max }}{2} \Gamma(\mathbf{q}) \lambda_{\mathbf{q}}\right| u .
$$

Thus, the optimal control is given by,

$$
\boldsymbol{u}=-\Gamma^{T}(\mathbf{q}) \lambda_{\mathbf{q}} /\left|\Gamma^{T}(\mathbf{q}) \lambda_{\mathbf{q}}\right|=\Gamma(\mathbf{q}) \lambda_{\mathbf{q}} /\left|\Gamma(\mathbf{q}) \lambda_{\mathbf{q}}\right|
$$

For the co-state boundary conditions one has,

$$
\lambda_{\mathbf{q}}\left(t_{f}\right)=\left.\frac{\partial \Phi\{\mathbf{q}(t)\}}{\partial \mathbf{q}}\right|_{t=t_{f}}=\mathbf{Q}_{f}\left(\mathbf{q}\left(t_{f}\right)-\mathbf{q}_{d}\right)
$$

Once the control is found from equation 2.12, equation 2.2 is used to define the angular velocity vector, which is then used to define the optimal input control torques. When 
implementing constraints, it is often more appropriate to use alternate representations of the quaternion such as the Euler vector and the Euler principal angle or the Gibbs vector.

\section{Optimal Translational Trajectory Synthesis}

The translational kinematics may be expressed as,

$$
\frac{d \mathbf{r}}{d t}+\boldsymbol{\Omega}(\boldsymbol{\omega}) \mathbf{r}=\mathbf{v}, \frac{d \mathbf{r}}{d t}=-\boldsymbol{\Omega}(\boldsymbol{\omega}) \mathbf{r}+\mathbf{v},
$$

where, $\boldsymbol{\Omega}(\boldsymbol{\omega})$ is defined in equation (2.1b). Hence, with,

$$
\begin{gathered}
\mathbf{A}_{\mathbf{r}}=\left[\begin{array}{cc}
-\boldsymbol{\Omega}(\omega) & 0 \\
0 & 0
\end{array}\right] \text { and } \mathbf{q}_{\mathbf{r}}=\left[\begin{array}{l}
\mathbf{r} \\
0
\end{array}\right], \\
\frac{d}{d t} \mathbf{q}_{\mathbf{r}}=\mathbf{A}_{\mathbf{r}} \mathbf{q}_{\mathbf{r}}+\left[\begin{array}{l}
\mathbf{v} \\
0
\end{array}\right] .
\end{gathered}
$$

Equation (3.3) is similar in form to the quaternion kinematics equation. It is often advantageous to use equations (3.3) rather than the dual quaternion formulation as the constraints are explicitly satisfied. In above equations, the linear velocity vector is treated as a control variable and expressed as,

$$
\mathbf{v}=|\mathbf{v}|_{\max } \boldsymbol{u},
$$

where the direction vector $\boldsymbol{u}$ is parametrized by two angles defining the direction of the $\mathbf{v}$ vector. Thus the direction vector $\boldsymbol{u}$ is expressed as,

$$
\boldsymbol{u}=\left[\begin{array}{lll}
\sin \alpha \cos \beta & \cos \alpha \cos \beta & \sin \beta
\end{array}\right]^{T} .
$$

When one is interested in the problem of finding the directional control,

$$
\boldsymbol{u}=\boldsymbol{u}(t), t_{0} \leq t \leq t_{f},
$$

the angular velocity direction time history is sought, such that it minimizes the cost functional:

$$
J=\left.0.5\left(\mathbf{r}(t)-\mathbf{r}_{d}\right)^{T} \mathbf{Q}_{r f}\left(\mathbf{r}(t)-\mathbf{r}_{d}\right)\right|_{t=t_{f}}=\left.\Phi_{r}\{\mathbf{r}(t)\}\right|_{t=t_{f}}{ }^{\prime}
$$

subject to, equations (3.1). Introducing the single state vector, $\mathbf{x}=\mathbf{r}$ so the equations (3.1), are expressed as,

$$
d \mathbf{x} / d t=\mathbf{f} .
$$

To solve the optimization problem, three Lagrangian multipliers or co-states are introduced given by the vector $\lambda_{\mathbf{r}}(t)$. Following Bryson and Ho (1969), a Hamiltonian function is defined as,

$$
H=-\lambda_{\mathbf{r}}^{T}(t) \boldsymbol{\Omega}(\boldsymbol{\omega}) \mathbf{r}(t)+\lambda_{\mathbf{r}}^{T}(t) \boldsymbol{u} .
$$

The corresponding differential equations that the co-state vector must satisfy are,

$$
\frac{d}{d t} \lambda_{\mathbf{r}}^{T}(t)=-\frac{d H}{d \mathbf{r}}=\lambda_{\mathbf{r}}^{T}(t) \Omega(\omega) .
$$

The optimality conditions for the control parameters are,

$$
\frac{\partial H}{\partial \alpha}=|\mathbf{v}|_{\max } \lambda_{\mathbf{r}}^{T}[\cos \alpha \cos \beta \quad-\sin \alpha \cos \beta \quad 0]=0,
$$

and 


$$
\frac{\partial H}{\partial \beta}=|\mathbf{v}|_{\max } \lambda_{\mathbf{r}}^{T}[-\sin \alpha \sin \beta \quad-\cos \alpha \sin \beta \quad \cos \beta]=0 .
$$

Hence it follows that the co-state vector $\lambda_{\mathbf{r}}(t)$, satisfies the relation,

$$
|\mathbf{v}|_{\max } \lambda_{\mathbf{r}}=\left.|\mathbf{v}|_{\max } \lambda_{\mathbf{r}}\left|\left[\begin{array}{lll}
\sin \alpha \cos \beta & \cos \alpha \cos \beta & \sin \beta
\end{array}\right]^{T}=\right| \mathbf{v}\right|_{\max } \lambda_{\mathbf{r}} \mid \boldsymbol{u}
$$

Thus, the optimal control is $\mathbf{v}=|\mathbf{v}|_{\max } \boldsymbol{u}, \boldsymbol{u}$ where is given by,

$$
\boldsymbol{u}=\lambda_{\mathbf{r}} /\left|\lambda_{\mathbf{r}}\right|
$$

For the co-state boundary conditions one has,

$$
\lambda_{\mathbf{r}}\left(t_{f}\right)=\left.\frac{\partial \Phi_{r}\{\mathbf{r}(t)\}}{\partial \mathbf{r}}\right|_{t=t_{f}}=\left.\mathbf{Q}_{r f}\left(\mathbf{r}(t)-\mathbf{r}_{d}\right)\right|_{t=t_{f}} .
$$

Once the control is found from equation 3.14, the attitude kinetics equations are used to define the angular velocity vector, which is then used to define the optimal input control torques.

\section{Translational Kinematics of an Aerospace Robotic Platform}

Consider a 3-1-3 sequence of Euler angles, where the angles are $\theta$ the longitude, a $90^{\circ}$ rotation about the " 1 " axis and $\phi$ the latitude. The corresponding transformations are,

$$
T_{3}(\theta)=\left[\begin{array}{ccc}
\cos \theta & \sin \theta & 0 \\
-\sin \theta & \cos \theta & 0 \\
0 & 0 & 1
\end{array}\right], T_{1}\left(90^{\circ}\right)=\left[\begin{array}{ccc}
1 & 0 & 0 \\
0 & 0 & 1 \\
0 & -1 & 0
\end{array}\right], T_{3}(\phi)=\left[\begin{array}{ccc}
\cos \phi & \sin \phi & 0 \\
-\sin \phi & \cos \phi & 0 \\
0 & 0 & 1
\end{array}\right] \text {. }
$$

The Earth-fixed to body fixed transformation is given by,

$$
T_{B E}=T_{3}(\theta) T_{1}\left(90^{\circ}\right) T_{3}(\phi), T_{B E}=\left[\begin{array}{ccc}
\cos \theta \cos \phi & \cos \theta \sin \phi & \sin \theta \\
-\sin \theta \cos \phi & -\sin \theta \sin \phi & \cos \theta \\
\sin \phi & -\cos \phi & 0
\end{array}\right] .
$$

Combined with the radial position $r, \theta$ the longitude and $\phi$ the latitude, constitute a set of spherical coordinates. The angular velocity components for the above 3-1-3 sequence are:

$$
\boldsymbol{\omega}=\left[\begin{array}{lll}
\dot{\theta} \sin \phi & \dot{\theta} \cos \phi & \dot{\phi}
\end{array}\right]^{T} .
$$

Now let $\gamma$ be the flight path angle, $\psi$ be the heading or yaw angle and $v$ the magnitude of the vehicle's velocity. Then the kinematic equations relating the velocity components to the rate of change of position in the spherical coordinates are,

$$
\dot{r}=v \sin \gamma, r \dot{\phi}=v \cos \gamma \sin \psi, r \cos \phi \dot{\theta}=v \cos \gamma \cos \psi .
$$

The equations (4.4) are non-singular as long as the variable $\phi$ satisfies $-\pi / 2<\phi<\pi / 2$; i. e. the vehicle does not overfly the poles.

\section{Translational Kinetics of an Aerospace Robotic Platform}

For completeness the translational kinetics of the platform orbiting the Earth in a low Earth orbit is also briefly presented. Consider first a non-rotating planet. The local acceleration due to gravitation, positive down, which is also the negative radial component may be defined as, 


$$
g=\mu / r^{2}
$$

The kinetic equations are expressed as,

$$
\begin{gathered}
\dot{v}=a_{s}-g \sin \gamma, \\
v \cos \gamma \dot{\psi}=a_{w}-\frac{v^{2}}{r} \cos ^{2} \gamma \cos \psi \tan \phi, \\
v \dot{\gamma}=a_{n}-\left(g-\frac{v^{2}}{r}\right) \cos \gamma, \\
\dot{m}=-T / v_{e},
\end{gathered}
$$

Equation (5.5) represents the rate of change of mass of the vehicle due to the expulsion of mass with relative velocity $v_{e}$ by the propulsion system. In equations (5.2) to (5.4) the aerodynamic forces in the tangential, normal and bi-normal directions are respectively defined by,

$$
m a_{s}=T \cos \alpha-D, m a_{w}=(L+T \sin \alpha) \sin \phi_{r}, m a_{n}=(L+T \sin \alpha) \cos \phi_{r} \text {. (5.6) }
$$

In equation 5.6, $\phi_{r}$ is the bank angle, $T$ is the body fixed thrust, $L$ is the aerodynamic lift which is linearly dependent on the elevator angle, $D$ is the aerodynamic drag, $\alpha$ is the angle of attack, which is equal to the pitch plus the flight path angles, $\alpha=\theta_{p}+\gamma$. The Euler angles defining the attitude of the vehicle are $\psi, \theta_{p}, \phi_{r}$ (yaw, pitch roll or 3-2-1 sequence) which can be obtained from the quaternion components. Given the Euler parameters or quaternion components,

$$
\eta=\cos (\phi / 2) ; \boldsymbol{\varepsilon}=\left[\begin{array}{lll}
\varepsilon_{1} & \varepsilon_{2} & \varepsilon_{3}
\end{array}\right]^{T}=\mathbf{n} \sin (\phi / 2),
$$

the transformation relating an orbiting reference frame to a body fixed frame is given by,

$$
\mathbf{T}_{B R}(\mathbf{q})=\left\{T_{i j}\right\}=\left[\begin{array}{ccc}
\eta^{2}+\varepsilon_{1}^{2}-\varepsilon_{2}^{2}-\varepsilon_{3}^{2} & 2\left(\varepsilon_{1} \varepsilon_{2}+\varepsilon_{3} \eta\right) & 2\left(\varepsilon_{1} \varepsilon_{3}-\varepsilon_{2} \eta\right) \\
2\left(\varepsilon_{1} \varepsilon_{2}-\varepsilon_{3} \eta\right) & \eta^{2}-\varepsilon_{1}^{2}+\varepsilon_{2}^{2}-\varepsilon_{3}^{2} & 2\left(\varepsilon_{2} \varepsilon_{3}+\varepsilon_{1} \eta\right) \\
2\left(\varepsilon_{1} \varepsilon_{3}+\varepsilon_{2} \eta\right) & 2\left(\varepsilon_{2} \varepsilon_{3}-\varepsilon_{1} \eta\right) & \eta^{2}-\varepsilon_{1}^{2}-\varepsilon_{2}^{2}+\varepsilon_{3}^{2}
\end{array}\right]
$$

Thus it follows that,

$$
\begin{gathered}
t=\operatorname{Trace}\left(T_{i j}\right)=3 \eta^{2}-\varepsilon_{1}^{2}-\varepsilon_{2}^{2}-\varepsilon_{3}^{2}=4 \eta^{2}-1 ; r=\sqrt{1+t}=2 \eta ; \eta=r / 2=\sqrt{1+t} / 2 ; \\
s=1 / 2 r=1 /(4 \eta) ; \varepsilon_{1}=\left(T_{23}-T_{32}\right) s, \varepsilon_{2}=\left(T_{31}-T_{13}\right) s, \varepsilon_{3}=\left(T_{12}-T_{21}\right) s .
\end{gathered}
$$

Hence the quaternion components may be related to the 3-2-1 Euler angle sequence components from the elements of the attitude transformation matrix.

In the case of a rotating planet with the atmosphere rotating with it, one has,

$$
\begin{gathered}
\dot{v}=a_{s}+g_{s}+c f_{v}, \dot{m}=-T / v_{e}, \\
v \cos \gamma \dot{\psi}=a_{w}+g_{w}-\frac{v^{2}}{r} \cos ^{2} \gamma \cos \psi \tan \phi+\left(c f_{\psi}+v \cos \gamma c o_{\psi}\right), \\
v \dot{\gamma}=a_{n}+\left(g_{n}+\frac{v^{2}}{r}\right) \cos \gamma+\left(c f_{\gamma}+v c o_{\gamma}\right) .
\end{gathered}
$$

The centrifugal ( $c f)$ and coriolis $(c o)$ terms due to the planets rotation rate $(\Omega)$ are: 


$$
\begin{gathered}
c f_{v}=\Omega^{2} r \cos \phi(\sin \gamma \cos \phi-\cos \gamma \sin \phi \sin \psi), \\
c f_{\psi}=-\Omega^{2} r \sin \phi \cos \phi \cos \psi, \\
c f_{\gamma}=-\Omega^{2} r \cos \phi(\cos \gamma \cos \phi+\sin \gamma \sin \phi \sin \psi), \\
c o_{\psi}=2 \Omega(\tan \gamma \cos \phi \sin \psi-\sin \phi), c o_{\gamma}=2 \Omega \cos \phi \cos \psi .
\end{gathered}
$$

\section{Optimal Translational Trajectory Synthesis for an Aerospace Robotic Platform}

Generally when constraints need to applied to the kinematic variables it is often convenient to express the kinematics in a frame where the constraints reduce to equality relations applied to the variables. In deriving the optimal control input, corresponding to equation 3.15 , for the translational kinematic equations in section 4 , equations 4.5 are often used in place of equation 3.1. Thus the Hamiltonian is defined by,

$$
H=\lambda_{r} v \sin \gamma+\lambda_{\theta} \frac{v \cos \gamma \cos \psi}{r \cos \phi}+\lambda_{\phi} \frac{v \cos \gamma \sin \psi}{r} .
$$

The differential equations that the co-states must satisfy are,

$$
\begin{gathered}
\dot{\lambda}_{r}=-\frac{\partial H}{\partial r}=\lambda_{\theta} \frac{v \cos \gamma \cos \psi}{r^{2} \cos \phi}+\lambda_{\phi} \frac{v \cos \gamma \sin \psi}{r^{2}}, \\
\dot{\lambda}_{\theta}=-\frac{\partial H}{\partial \theta}=0, \dot{\lambda}_{\phi}=-\frac{\partial H}{\partial \phi}=-\lambda_{\theta} \frac{v \cos \gamma \cos \psi}{r(\cos \phi)^{2}} \sin \phi .
\end{gathered}
$$

The control input is expressed in terms of the fixed magnitude $v=|\mathbf{v}|_{\max }$ and the direction of the velocity vector. Thus the optimality conditions for the control parameters are,

$$
\begin{gathered}
\frac{\partial H}{\partial \gamma}=\lambda_{r} v \cos \gamma-\lambda_{\theta} \frac{v \sin \gamma \cos \psi}{r \cos \varphi}-\lambda_{\varphi} \frac{v \sin \gamma \sin \psi}{r}=0, \\
\frac{\partial H}{\partial \psi}=-\lambda_{\theta} \frac{v \cos \gamma \sin \psi}{r \cos \varphi}+\lambda_{\varphi} \frac{v \cos \gamma \cos \psi}{r}=0 .
\end{gathered}
$$

Hence co-states satisfy the relation,

$$
v\left[\begin{array}{lll}
\lambda_{r} & \lambda_{\theta} & \lambda_{\varphi}
\end{array}\right]=\mid v\left[\begin{array}{lll}
\lambda_{r} & \lambda_{\theta} & \lambda_{\varphi}
\end{array}\right]\left[\begin{array}{lll}
\sin \gamma & r \cos \phi \cos \gamma \cos \psi & r \cos \gamma \sin \psi
\end{array}\right] .
$$

Thus, the optimal control parameters defining the direction of the velocity vector are given by,

$$
\left[\begin{array}{lll}
\sin \gamma & \cos \gamma \cos \psi & \cos \gamma \sin \psi
\end{array}\right]^{T}=\left[\begin{array}{ccc}
1 & 0 & 0 \\
0 & r \cos \phi & 0 \\
0 & 0 & r
\end{array}\right]^{-1} \frac{\left[\begin{array}{ccc}
\lambda_{r} & \lambda_{\theta} & \lambda_{\varphi}
\end{array}\right]^{T}}{\mid\left[\begin{array}{lll}
\lambda_{r} & \lambda_{\theta} & \lambda_{\varphi}
\end{array}\right]} .
$$

For the co-state boundary conditions one has,

$$
\lambda_{r}\left(t_{f}\right)=\left.\left(r(t)-r_{d}\right)\right|_{t=t_{f}}, \lambda_{\theta}\left(t_{f}\right)=\left.\left(\theta(t)-\theta_{d}\right)\right|_{t=t_{f}}, \lambda_{\phi}\left(t_{f}\right)=\left.\left(\phi(t)-\phi_{d}\right)\right|_{t=t_{f}} .
$$




\section{Extension to an On-board Robot Manipulator}

In the presence of an on-board manipulator, to be able compute the trajectories of the manipulator links, it is essential to include the joint kinematics. In general, it is possible to assume that the manipulator joints have a single degree of freedom and are either revolute or prismatic joints. Under these circumstances, in many situations, the joint kinematics are given by scalar, uncoupled equations of the form, $\dot{\theta}_{i}=\omega_{i}$ for the $i^{\text {th }}$ revolute joint and $\dot{d}_{i}=v_{i}$ for the $i^{\text {th }}$ prismatic joint. Following Yakimenko (2000), the optimal trajectories in these cases are expressed by eighth order polynomials in a scaled arc-length parameter where the coefficients are determined by the maximum bounds on the jerk, jerk-rate, acceleration and velocity as well as boundary conditions on the scale factor, position and acceleration at the two end points. The method has been adapted also by Cowling, Whidborne and Cooke (2006), Lukacs and Yakimenko (2007), Etchemendy (2007) and is discussed in some detail by Vepa (2016) in section 14.13 . The method integrating such a trajectory segment into the complete motion plan is discussed by Dugar, Choudhury and Scherer (2017).

Once the velocity profile over a time frame is known from the co-state equations and the boundary conditions, the optimum forces and torques acting on the vehicle as well as the optimum torques acting the manipulator joints may be determined.
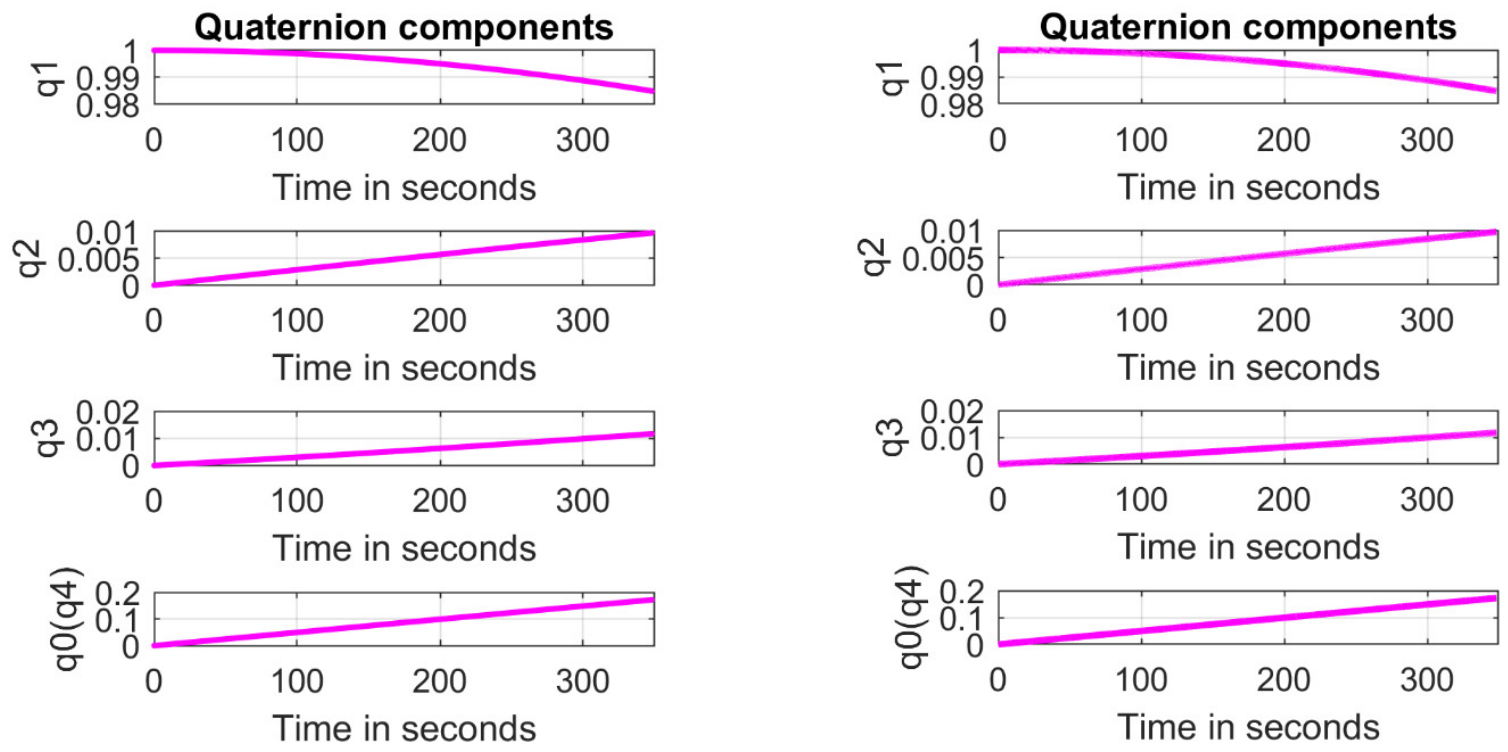

Fig. 1 The synthesized quaternion reference trajectory

Fig. 2 The actual quaternion trajectory tracked by the vehicle

\section{Typical Simulation Examples}

The first example considered is one where the quaternion attitude needs to smoothly change from its current initial value $\left(\eta=0, \varepsilon=\left[\begin{array}{lll}1 & 0 & 0\end{array}\right]^{T}\right)$ to final desired value, which involves a small but high precision change in the attitude. Figure 1 illustrates the synthesized trajectory while figure 2 illustrates the actual quaternion trajectory components as a space vehicle tracks the reference trajectory. In the figure $1, \eta$ is shown as $q_{0}\left(q_{4}\right)$ while $\boldsymbol{\varepsilon}=\left[\begin{array}{lll}q_{1} & q_{2} & q_{3}\end{array}\right]^{T}$. The 
estimated maximum error between the plots in figures 1 and 2 is less than $3 \%$ always. Figure 3 illustrates a typical set angular velocity time histories required to achieve the desired attitude.
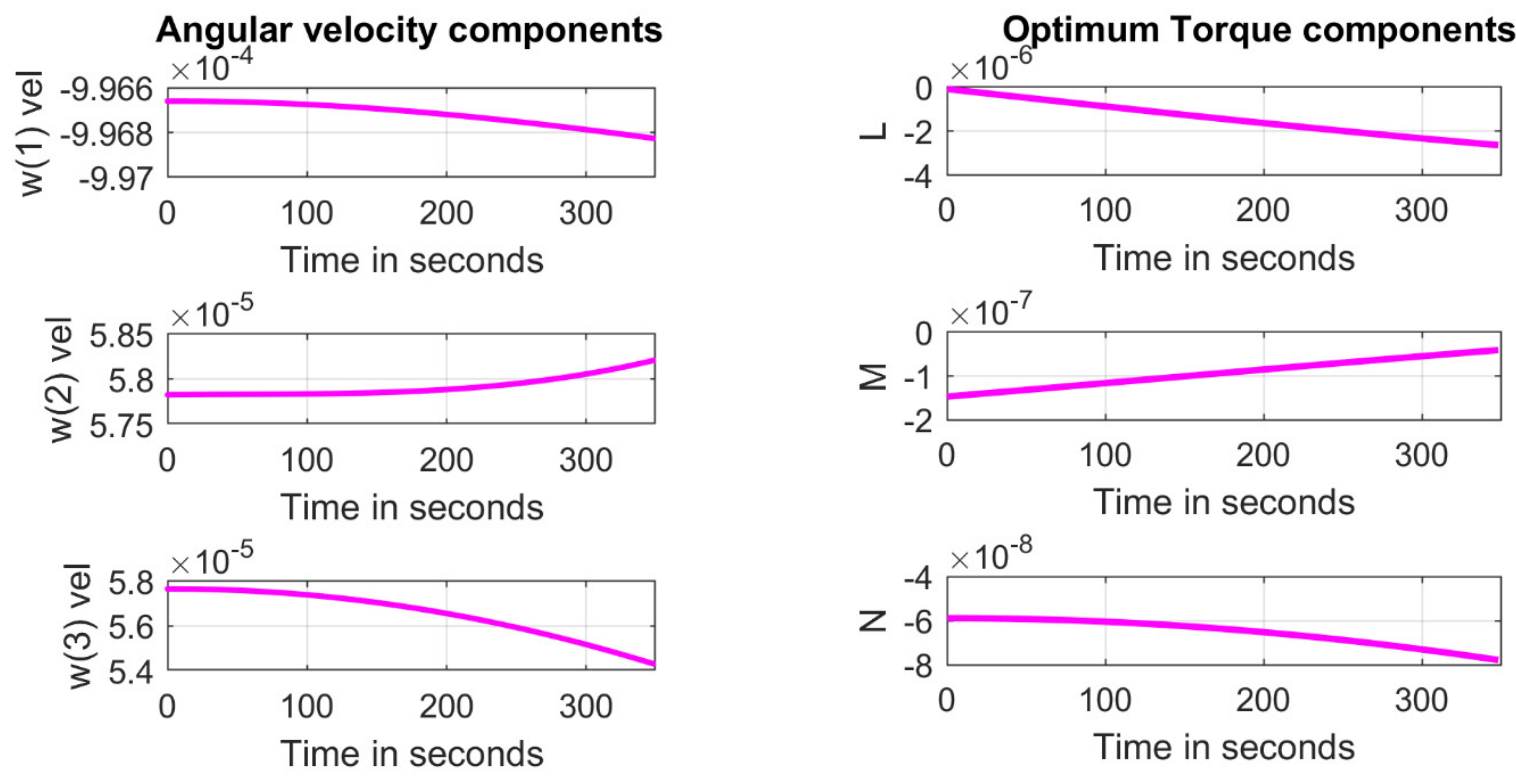

Fig 3 The corresponding reference angular velocity time histories

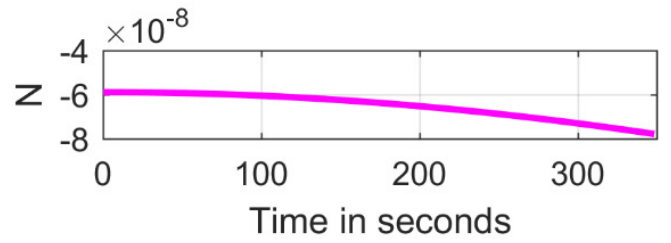

Fig. 4 The corresponding torque (in Nm) time histories
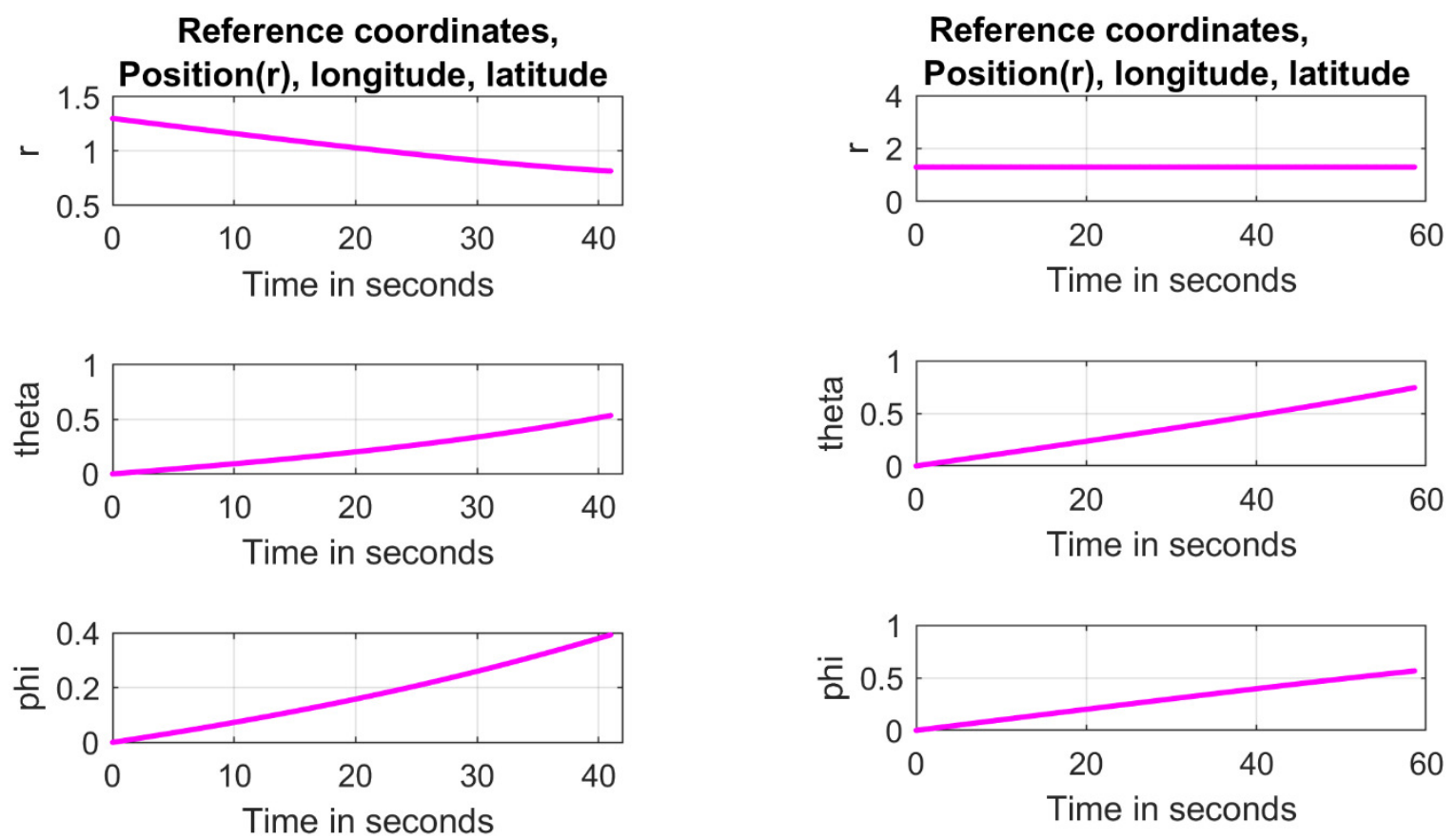

Fig 5 The time histories of the position longitude and latitude

Fig. 6 The time histories of the position, which is fixed, longitude and latitude 
In figure 4 are shown the corresponding torques, including the gravity gradient torques for a typical CUBESAT type space vehicle. The torques are provided by an electric magnetic actuation system. The two-point boundary value problem was solved by using the MATLAB $\mathrm{m}$-file, bvp4c.m. The maximum magnitude of the angular velocity is restricted to $0.001 \mathrm{rad} / \mathrm{s}$. When this method is applied to a robotic manipulator with three or more degrees of freedom, once the reference quaternion time histories are obtained, the joint angles are found from the solutions for the inverse kinematics.

The second example considered defines the trajectory of a low Earth orbiting vehicle. The vehicle translational kinematics is defined by equations 4.4 to 4.6 . In figure 5 are shown the position or altitude, longitude and latitude as it travels towards a destination longitude and latitude while slowly losing altitude. In figure 6 are shown the position or altitude, longitude and latitude as it travels towards a destination longitude and latitude while holding the altitude fixed. To achieve the fixed altitude the corresponding co-state variable is set equal to zero. In both these figures the altitude is normalized and the initial altitude is set at 1.3.

The final example is a UAV such as a quadcopter which is modelled as a body rather than as a point mass. The attitude kinematics continues to be given by equations 2.1 ,

$$
\frac{d \mathbf{q}}{d t}=\frac{1}{2} \mathbf{A}_{\boldsymbol{\omega}}(\boldsymbol{\omega}) \mathbf{q}=\frac{1}{2} \Gamma(\mathbf{q}) \boldsymbol{\omega} .
$$

The position kinematics is given by,

$$
\dot{\mathbf{r}}=\mathbf{T}_{B I}^{T}(\mathbf{q}) \mathbf{v}_{b},
$$

where $\mathbf{v}_{b}=\left[\begin{array}{lll}u & v & w\end{array}\right]^{T}$ is the velocity of the vehicle in body fixed coordinates while $\mathbf{T}_{B I}(\mathbf{q})$ is the transformation from an inertial of space fixed frame to the body fixed frame. It may be expressed in terms of the components of the quaternion vector.

The Hamiltonian in this case is:

$$
\begin{gathered}
H=\lambda_{\mathbf{q}}^{T} \frac{1}{2} \mathbf{A}_{\boldsymbol{\omega}}(\boldsymbol{\omega}) \mathbf{q}+\lambda_{\mathbf{r}}^{T} \mathbf{T}_{B I}^{T}(\mathbf{q}) \mathbf{v}_{b}=\lambda_{\mathbf{q}}^{T} \frac{1}{2} \Gamma(\mathbf{q}) \boldsymbol{\omega}+\lambda_{\mathbf{r}}^{T} \mathbf{T}_{B I}^{T}(\mathbf{q}) \mathbf{v}_{b}, \\
\frac{d}{d t} \lambda_{\mathbf{q}}^{T}(t)=-\frac{\partial H}{\partial \mathbf{q}}, \frac{d}{d t} \lambda_{\mathbf{r}}^{T}(t)=-\frac{\partial H}{\partial \mathbf{r}}=0 .
\end{gathered}
$$

Thus one may choose, $\lambda_{\mathbf{r}}^{T}(t) \equiv 0$ and $\mathbf{v}_{b}=\mathbf{v}_{b, \max }=\left[\begin{array}{lll}u_{\max } & v_{\max } & w_{\max }\end{array}\right]^{T}$ is a constant vector. Hence, it follows that,

$$
\frac{d}{d t} \lambda_{\mathbf{q}}^{T}(t)=-\frac{\partial H}{\partial \mathbf{q}}=-\frac{1}{2} \lambda_{\mathbf{q}}^{T} \mathbf{A}_{\boldsymbol{\omega}}(\boldsymbol{\omega}) .
$$

The angular velocity vector is treated as a control variable and expressed as, $\omega=|\omega|_{\max } \boldsymbol{u}$, where $\boldsymbol{u}$, may continue to be chosen in accordance with equation 2.12. Thus the problem reduces to one where the optimal attitude variation only need to be computed using the methodology outlined in section 2. For the translational trajectories, in the body frame a relatively simple approach is to relate the velocity vector to the applied specific thrust vector (thrust vector per unit mass) and the net drag or lift forces. Ignoring the gravity forces, the equation relating the velocity vector $\mathbf{v}_{b}$, to the specific thrust magnitude and to the position vector in the body frame $\mathbf{r}_{b}$, are assumed to be given by, 


$$
\dot{\mathbf{v}}_{b}+\boldsymbol{\omega} \times \mathbf{v}_{b}=T \mathbf{u}-\mathbf{K}_{l d} . \times \mathbf{v}_{b} . \times \mathbf{v}_{b}, \dot{\mathbf{r}}_{b}+\boldsymbol{\omega} \times \mathbf{r}_{b}=\mathbf{v}_{b},
$$

where $T$ is the magnitude of the specific thrust, $\mathbf{K}_{l d}$ is a vector of constants relating the lift or drag to the square of the velocity component and '. $x$ ' is the elementwise product of two vectors. The reference velocity and reference position components may then be obtained with $\boldsymbol{\omega}=\mathbf{0}$ in steady state. Typical non-dimensional plots of the position and velocity components in the body frame obtained in this way are shown in figure 7 and 8 respectively. They may then be transformed to the space fixed frame by using the transformation $\mathbf{T}_{B I}(\mathbf{q})$ obtained from the reference quaternion components. In this case a model of the vehicle dynamics is used and the trajectory synthesis is not purely based on kinematic relations.
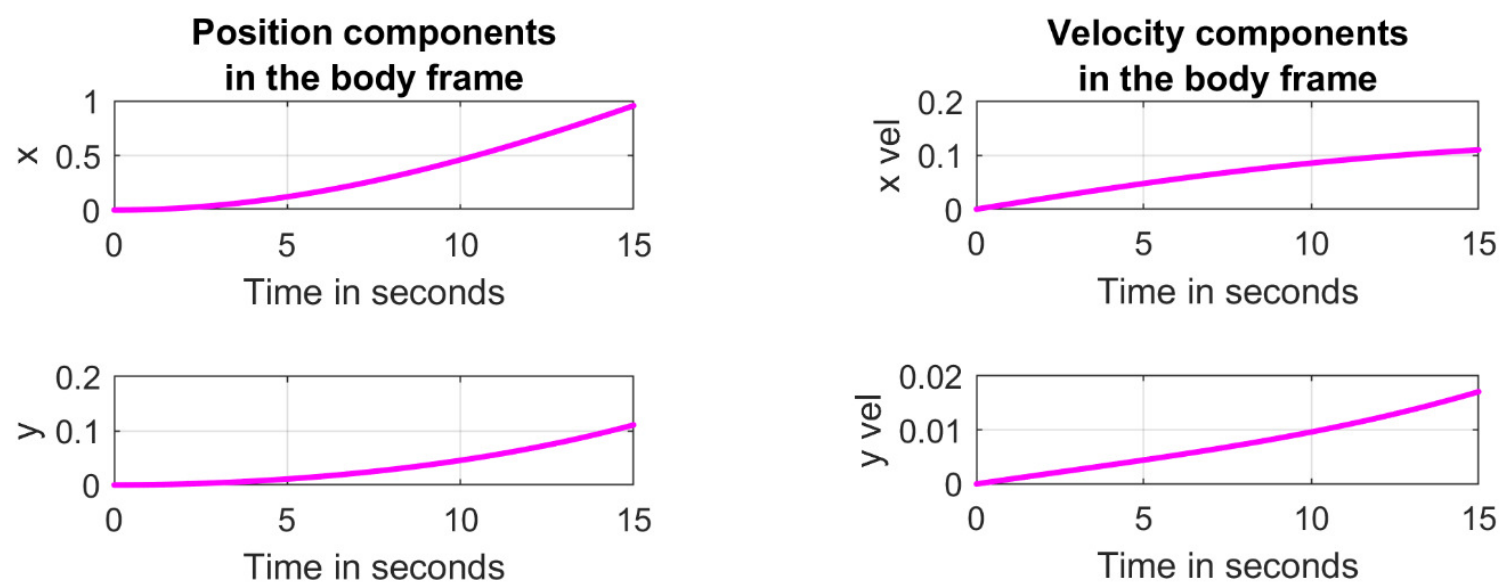

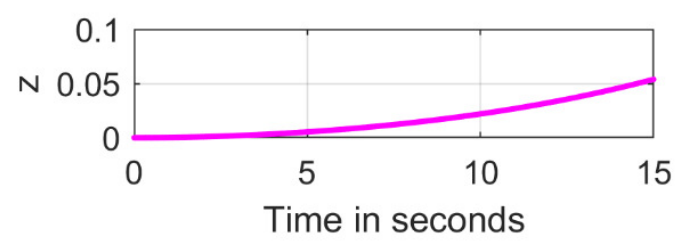

Fig 7 The time histories of the position components in the body frame

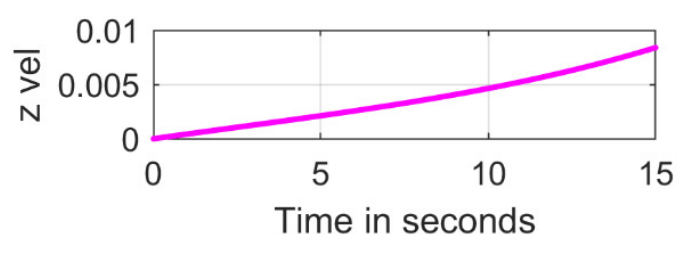

Fig. 6 The time histories of the velocity components in the body frame.

\section{Discussion and Conclusions}

In this work the feasibility of using a quaternion formulation of the attitude kinematics to develop the attitude acquisition trajectory using small changes in the angular velocity components has been successfully demonstrated. The application of the methodology to the translational kinematics is also demonstrated. The usefulness of this work is in its application to space robotic manipulators where the change in pose can be defined as a set of sequential changes in the attitudes of serially connected links with several revolute and some prismatic joints. Although the change in pose may be conveniently defined by a set of dual quaternions, the actual attitude acquisition problem is formulated as a sequence of vector attitude optimal synthesis problems similar to the form considered in this paper. When a set of manipulator links are serially connected with only revolute joints, the synthesis problem reduces to the simultaneous synthesis of attitude time histories of these links. When a finite number of prismatic joints are present, the problem reduces to a set of independent synthesis problems. 
The complete pose is found by accumulating the attitudes with alternate representations of the other vectors. It is demonstrated succinctly that the methodology may be applied, with minimal use of the dynamics, to actual space and aerial robotic manipulators attached to a vehicle in flight, a planetary rover (Vepa, 2019) or a robotic vehicle, where the attitude of the vehicle and the pose of the manipulator links or vehicle itself must be obtained. Apart from the simplicity, the fact they are non-dimensional implies that they could be used in variety of different physical solutions, by appropriate re-scaling.

\section{References}

Bryson, A. E. Jr. and Ho Y. C., Applied Optimal Control. Ginn \& Co., Waltham, MA, (1969).

Cowling, I. D., Whidborne, J. F., and Cooke, A. K., Optimal Trajectory Planning and LQR Control for a Quadrotor UAV, Proc. of the International Conference Control-2006, Glasgow, Scotland, August 30 - September 11, (2006).

Dugar, V., Choudhury, S., and Scherer, S., A кITE in the Wind: Smooth Trajectory Optimization in a Moving Reference Frame, 2017 IEEE International Conference on Robotics and Automation (ICRA), 109-116, (2017).

Etchemendy, M., Flight Control and Optimal Path Planning for UAVs, MSc Thesis, School of Engineering, Cranfield University, August, (2007).

Filipe, N. and Tsiotras, P., Adaptive Position and Attitude-Tracking Controller for Satellite Proximity Operations Using Dual Quaternions. J. Guid. Control Dyn. 38(4), 566-577, (2014).

Lagache M-A., Serres, U. and Andrieu, V., Minimal time synthesis for a kinematic drone model. Mathematical Control and Related Fields, AIMS, 7 (2), 259 - 288, (2017).

Lukacs, J. A, Yakimenko, O. A, Trajectory-Shape-Varying Missile Guidance for Interception of Ballistic Missiles during the Boost Phase, AIAA Guidance, Navigation and Control Conference and Exhibit, Hilton Head, South Carolina, August 20-23, (2007).

Özgür, E. and Mezouar, Y. Kinematic Modeling and Control of a Robot Arm Using Unit Dual Quaternions. Robot. Autom. Syst., 77, 66-73, (2016).

Sjøberg A. M. and Egeland, O., Kinematic Feedback Control Using Dual Quaternions, Proc. of the 26th Mediterranean Conference on Control and Automation, (IEEE), Zadar, Croatia, June 19-22, 2018, DOI: 10.1109/MED.2018.8442800.

Valverde A. and Tsiotras, P., Spacecraft Robot Kinematics Using Dual Quaternions, Robotics (MDPI) 7(64), 1-24, doi:10.3390, (2018).

Vepa, R., Nonlinear Control of Robots and Unmanned Aerial Vehicles: An Integrated Approach, CRC Press, Boca Raton, FL. USA, (2016).

Vepa, R., Dynamics and Control of Autonomous Space Vehicles and Robotics, Cambridge University Press, New York: NY, USA (2019).

Vukobratovic, M. and Kircanski, M., Kinematics and Trajectory Synthesis of Manipulation Robots, Springer Science \& Business Media, (2013).

Wolek, A., Cliff, E. M. and Woolsey, C. A. Time-Optimal Path Planning for a Kinematic Car with Variable Speed, J. Guid. Control Dyn. 39(10) 2374-2390, (2016).

Yakimenko, O. A, Direct method for rapid prototyping of near-optimal aircraft trajectories, $J$. Guid. Control Dyn. 23(5), (2000). 